\title{
Historia
}

\section{La historia por escribir en los Colegios Oficiales de Enfermería provinciales, los archivos como fuentes de investigación}

\author{
The story by writing to the provincial Colleges of Nursing, \\ archives and research sources
}

\section{A história por escrito às Faculdades Provinciais de Profissionais de Enfermagem, arquivos como fontes de pesquisa}

Diego José Feria Lorenzo ${ }^{1}$ y José Eugenio Guerra González ${ }^{2}$.

${ }^{1}$ Diplomado en Enfermería y Licenciado en Historia Profesor Titular de la Facultad de Enfermería de la Universidad de Huelva ${ }^{2}$ Diplomado en Enfermería. Servicio de Urgencias del Hospital Virgen de Valme, Alcalá de Guadaira, Sevilla.

Correo electrónico: ppguerra30@hotmail.com

Cómo citar este artículo en edición digital: Feria Lorenzo, D.J. y Guerra González, J.E. (2016). La historia por escribir en los Colegios Oficiales de Enfermería provinciales, los archivos como fuentes de investigación.. Cultura de los Cuidados (Edición digital),20(45). Disponible en: <http://dx.doi.org/10.14198/cuid.2016.45.04.>

Correspondencia: Diego José Feria Lorenzo. Facultad de Enfermería de la Universidad de Huelva, Avda. Tres de marzo s/n Huelva. CP 21071

Correo electrónico: diego.feria@denf.uhu.es

Recibido: 07/012/2015; Aceptado: 5/06/2016

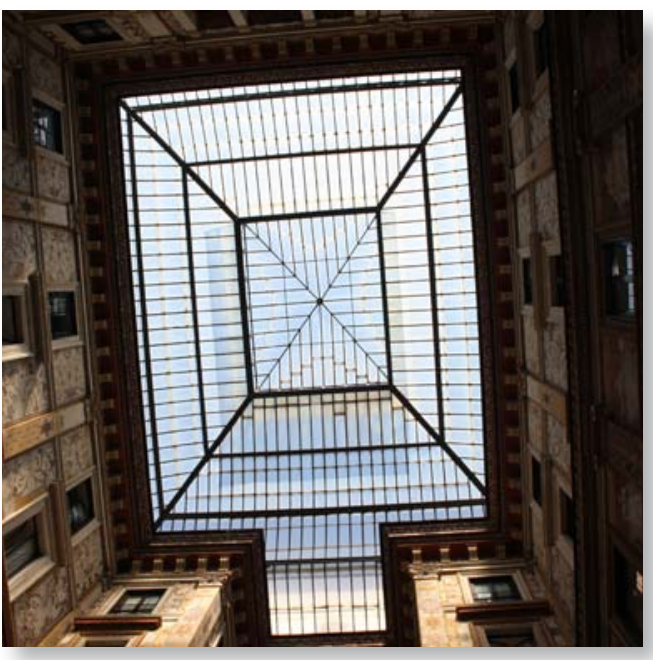

ABSTRACT

Research on Nursery History has a wide range of sources at its disposal. We must still investigate documents kept in professional colleges, as they are an accurate reflection of care and caregiver in the period of scientific development of the discipline. In this paper we shall describe the process of reorganization in the archive of the Nursery College in Huelva, as well as the situation of most Spanish nursery archives. Their classification aims to serve the College administration, which generates them, as well as the professionals and research, cultural diffusion and the reconstruction of this period of Nursery History.

Keywords: archives, historical sources of nursing, documentation in nursing.

\section{RESUMO}

Pesquisas em história da enfermagem temum grande número de fontes. Inexplorada é a documentação existente nas escolas profissionais, um verdadeiro reflexo de cuidados 
e cuidadores na fase do desenvolvimento profissional e científica da disciplina. Com esta comunicação pretende-se descrever, por um lado, o processo de organização que estamos realizando no arquivocom o Huelva enfermeira da escola; e em segundo lugar, a situação oferecido em organização de seus arquivos o restante deste instituições provinciais espanhóis. A classificação destes fundos é feito para o serviço da administração da faculdade é o corpo que gera, para os profissionais e, especialmente, para a pesquisa, difusão cultural e construção desse período na história da enfermagem.

Palavras-chave: processos, arquivos, histórico fontes de enfermagem, documentação de enfermagem.

\section{RESUMEN}

La investigación en historia de la enfermería cuenta con un amplio número de fuentes. Aún por explorar está la documentación existente en los colegios profesionales, fiel reflejo de los cuidados y cuidadores en la etapa de la profesionalización y el desarrollo científico de la disciplina. Con este artículo pretendemos describir, por un lado, el proceso de organización que estamos llevando a cabo en el archivo del colegio de enfermería de Huelva; y por otro, la situación que ofrecen en cuanto a organización de sus archivos el resto de estas instituciones provinciales españolas. La clasificación de esos fondos se realiza para el servicio de la administración colegial que es el órgano que la genera, para los profesionales y sobre todo para la investigación, la difusión cultural y la construcción de ese periodo de la historia de enfermería.

Palabras Clave: archivos, archivística, fuentes históricas de enfermería, documentación en enfermería.

\section{INTRODUCCIÓN}

La investigación en Historia de la Enfermería cuenta con un amplio y relevante número de fuentes que nos proporcionan instituciones sanitarias de práctica clínica, yacimientos arqueológicos, archivos, bibliotecas, etc. Aún por explorar está la amplia documentación que existe en los colegios profesionales, la referida a los últimos cien o ciento veinticinco últimos años en España. En la mayoría de ellos está latente una información que aún está por descubrir y que es fiel reflejo de los cuidados y cuidadores en la etapa de la profesionalización y el desarrollo científico de nuestra disciplina. En este periodo, los colegios han desarrollado un papel en muchos casos fundamental para la consolidación profesional.

Por la relevancia que ésta documentación tiene para la construcción de nuestra historia profesional queremos concienciar de su puesta al día, pues en muchos colegios parte de ella se ha perdido, eliminada como "papeles viejos". Otra parte se puede localizar apilada, quizás deteriorándose, en algún rincón de alguna dependencia de estas instituciones, y por la buena voluntad de algún enfermero que la ha mantenido a la espera de que alguien pueda organizarla en un futuro, está en un lugar que no reúne las condiciones idóneas para su conservación.

Es este el fin principal de toda organización archivística documental, la conservación, y junto a él la recuperación y la difusión como objetivos últimos de la misma. Para la realización de la ordenación de los archivos colegiales es necesario contar con el conocimiento y aplicación de una metodología archivística. Ésta parte inicialmente del "principio de procedencia”, que establece que los fondos han de conservarse y respetarse según la estructuración y orden original. Para consolidar este 
principio el conocimiento de la institución y su funcionamiento es fundamental, así como sus métodos de trabajo y evolución a lo largo del tiempo, lo que facilita la creación de un buen cuadro de clasificación. Implícita en esta premisa se encuentra el segundo nivel en la teoría archivística, el "principio de respeto al orden o estructura interna de los documentos". Los fondos del archivo deben contar con la clasificación correspondiente a las estructuras administrativas del organismo que lo ha generado. El conocimiento de la legislación referida a la organización colegial y la historia de la enfermería en España se hacen necesarios para la correcta organización de estos fondos. No nos podemos olvidar que los inicios o intentos de las asociaciones de profesionales sanitarios surgen a partir de mediados del XIX, con la reorganización de las profesiones sanitarias a partir de la Ley General de Sanidad de 1855 y de la Ley Moyano de Instrucción Pública de 1857. Será en la segunda mitad de este siglo cuando los primeros colegios de practicantes inicien su andadura en algunas provincias, entre las que podemos citar a Madrid en 1863, Sevilla en 1864 y Barcelona en 1866 (Expósito González, 2010, p. 30) como las pioneras en todo el territorio español. La obligatoriedad de la colegiación para el desempeño de la práctica profesional de practicantes será en 1929 y la de matronas en 1930.

La participación de los profesionales de enfermería en la clasificación de esta documentación se hace necesaria, y sobre todo, aquellos que conocen la historia disciplinar. Nuestra intención en este sentido pretende describir, por un lado, el proceso de organización que estamos llevando a cabo en el Archivo del Colegio de Enfermería de Huelva; y por otro, la situación que ofrecen en cuanto a organi- zación de sus archivos históricos el resto de los colegios provinciales españoles.

La información requerida de los colegios provinciales de enfermería se ha recogido a través de contactos telefónicos y de correos electrónicos enviados a los presidentes de estas instituciones. Se elaboró una carta tipo en la que se pedía, información sobre la fecha de fundación, si el archivo histórico del organismo en cuestión estaba organizado, y por último, si existía alguna publicación sobre la historia del Colegio. Algunos de ellos al celebrar el centenario de creación institucional han elaborado un libro donde se recoge el devenir histórico de la institución, caso del colegio de Sevilla, Teruel y Gipuzkoa, e incluso algunos han financiado la publicación de la historia de la enfermería provincial, como es el caso de Álava ${ }^{1}$.

\section{DESARROLLO DEL TEMA}

Los Archivos provinciales de los Colegios de Enfermería de España.

La información demandada para conocer la situación de la organización archivística y de fundación de los colegios provinciales españoles fue registrada en una tabla de Excel, de la que hemos extraído unos cuadros en los que la podemos observar de forma global. Tanto en la Tabla I como en la Tabla II, la información conseguida, en líneas generales es muy escasa, debido a la ausencia de contestación de los organismos objeto de estudio. Aunque es de destacar que algunos nos han enviado copias de las Actas Fundacionales, como es el caso de la del Colegio de Segovia, editada con objeto de la celebración del centenario (Figura I). 
FIGURA I: ACTA FUNDACIONAL DEL COLEGIO DE SEGOVIA.
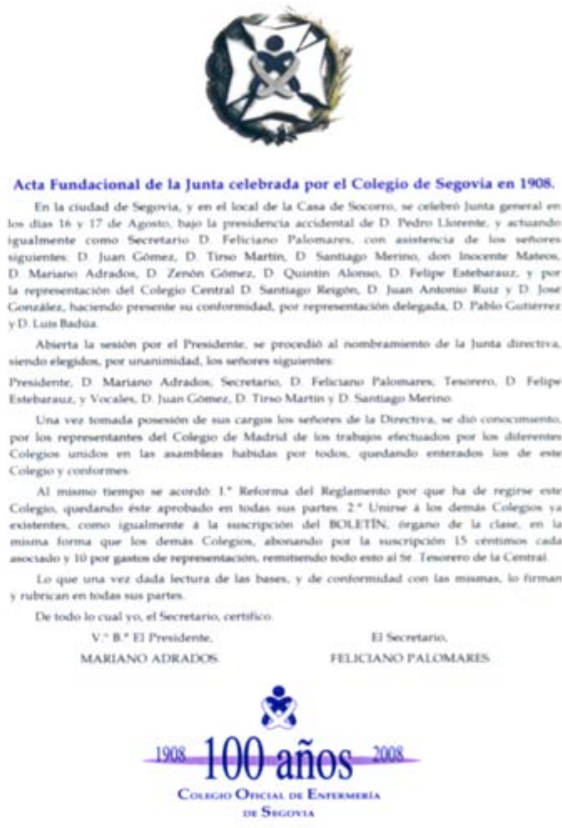

Fuente: Colegio Oficial de Enfermería de Segovia.
Otras referencias que aparecen en las tablas es fruto de la búsqueda bibliográfica en obras citadas en este texto y en los libros editados en los centenarios, información que en muchos casos no está bien contrastada, ya que podemos encontrar varias fechas en la fundación de muchos colegios provinciales. La creación de organismos de esta índole en poblaciones que no eran la capital provincial, en fechas tempranas, es otro de los hechos que hemos constatado, aunque no podemos avanzar si fueron asimilados a ellos posteriormente, como es el caso del de San Fernando en Cádiz (Tabla I). La pertenencia al de otra provincia limítrofe es otro de los factores que podemos encontrar en otros, o en una fundación tardía en el tiempo con respecto a la generalidad de la fecha de inicio del resto de estas instituciones.

Si extraemos los datos de fundación y realizamos una representación como la del Gráfico I, observamos que el mayor número de fundaciones colegiales (15), datan del periodo de

TABLA I: COLEGIOS DE LA MITAD SUR PENINSULAR.

\begin{tabular}{|c|c|c|c|c|}
\hline C. Aut noma & Provincia & H. Colegio & Fundaci n/centenario & Archivo \\
\hline \multirow[t]{8}{*}{ Andalue $\mathbf{a}$} & Almer $\mathbf{a}^{-}$ & $\mathrm{Si}$ & 1885 & \\
\hline & $\mathrm{C} \square \mathrm{diz}$ & No & $08 / 12 / 1900 \square 1901$ & \\
\hline & $\mathrm{C} \sqsubset$ rdoba & No & 1886 & \\
\hline & Granada & No & 1927 & \\
\hline & Huelva & No & 1912 & No \\
\hline & $\mathrm{Ja} \cap \mathrm{n}$ & No & $03 / 04 / 1913$ & \\
\hline & M laga & No & $27 / 3 / 1897$ & \\
\hline & Sevilla & $\mathrm{Si}$ & $17 / 7 / 1864$ & No \\
\hline \multirow[t]{2}{*}{ Extremadura } & $\mathrm{C} \subset$ ceres & No & & \\
\hline & Badajoz & No & & \\
\hline \multirow[t]{5}{*}{ Castilla/La Mancha } & Albacete & No & & \\
\hline & Ciudad Real & No & $21 / 3 / 1913$ & \\
\hline & Cuenca & No & & \\
\hline & Guadalajara & No & & \\
\hline & Toledo & No & & \\
\hline Murcia & Murcia & No & 1910 & \\
\hline Madrid & Madrid & $\mathrm{Si}$ & $1863 / 1902$ & No \\
\hline \multirow[t]{3}{*}{ Valencia } & Alicante & & & \\
\hline & Valencia & & 1903 & \\
\hline & Castell $\sqsubset \mathrm{n}$ & & & \\
\hline \multirow[t]{2}{*}{ Canarias } & Tenerife & No & 1912 & \\
\hline & Gran Canaria & & & \\
\hline Ceuta & Ceuta & No & 1914 & \\
\hline Melilla & Melilla & & 1924 & \\
\hline San Fernando & $\mathrm{C} \square \mathrm{diz}$ & & 1922 & \\
\hline
\end{tabular}

Fuente: Organizaciones colegiales provinciales y bibliograf $a$ referenciada. Elaboraci $\lceil$ n Propia. 
TABLA II: COLEGIOS DE LA MITAD NORTE PENINSULAR.

\begin{tabular}{|c|c|c|c|c|}
\hline C. Aut noma & Provincia & H. Colegio & Fundaci $\square$ /centenario & Archivo \\
\hline \multirow[t]{9}{*}{ Castilla/Le $\square$ n } & vila & No & $27 / 09 / 1904$ & No \\
\hline & $\mathrm{Le} \sqcap \mathrm{n}$ & No & & \\
\hline & Soria & No & 1899 & No \\
\hline & Salamanca & No & $1976 ?$ & No \\
\hline & Segovia & No & 1908 & No \\
\hline & Valladolid & No & $05 / 09 / 1905$ & No \\
\hline & Zamora & No & & \\
\hline & Palencia & No & 1909 & No \\
\hline & Burgos & No & desconocida & No \\
\hline Cantabria & Cantabria & No & $1925 / 1937$ & $\mathrm{Si}$ \\
\hline Asturias & Oviedo & Elabor ndose & & \\
\hline \multirow[t]{3}{*}{ Pas Vasco } & Guip zcoa & $\mathrm{Si}$ & $27 / 07 / 1904$ & No \\
\hline & lava & $\mathrm{Si}$ & $04 / 03 / 1902$ & \\
\hline & Bizkaia & No & & \\
\hline \multirow[t]{4}{*}{ Catalu $\square \mathbf{a}$} & Barcelona & & $1866 / 1884$ & \\
\hline & Tarragona & & & \\
\hline & Gerona & No & 1925 & No \\
\hline & Lleida & & & \\
\hline \multirow[t]{3}{*}{ Arag $\sqrt{n}$} & Huesca & No & 1917 & No \\
\hline & Zaragoza & & $1884 / 1899$ & \\
\hline & Teruel & $\mathrm{Si}$ & 1909 & No \\
\hline Navarra & Navarra & & 1899 & \\
\hline La Rioja & Logro-o & $\mathrm{Si}$ & 1899 & No \\
\hline \multirow[t]{4}{*}{ Galicia } & A Coru-a & & $1865 / 1906$ & \\
\hline & Lugo & & & \\
\hline & Ourense & & & \\
\hline & Pontevedra & & & \\
\hline Islas Baleares & Mallorca & & & \\
\hline
\end{tabular}

Fuente: Organizaciones colegiales provinciales y bibliograf a referenciada. Elaboraci $\llbracket n$ Propia.

1901 a 1920, antes de 1900 fueron 13, y posterior a $1920,{ }^{5}$.

La organización de estos archivos históricos en la mayoría de los casos está ausente o bien se confunde con la creación de un museo de piezas, utensilios u objetos sanitarios, como es el caso del que aparece en una página web de un colegio provincial:

El Colegio Oficial de Enfermería [...] tiene un Archivo Histórico de la profesión, en particular, y de la sanidad en general que ha ido tomando cuerpo tras las peticiones de nuestra institución para que aquellas personas o entidades que deseen donar piezas, utensilios, libros $u$ objetos de claro significado sanitario, lo entreguen al Colegio al objeto de ir configurando un Archivo donde las generaciones futuras puedan conocer de primera mano cuáles eran sus herramientas $y$, en consecuencia, procedimientos, en los procesos de atención al ser humano ${ }^{2}$.

\section{GRÁFICO I: NÚMERO DE FUNDACIONES}

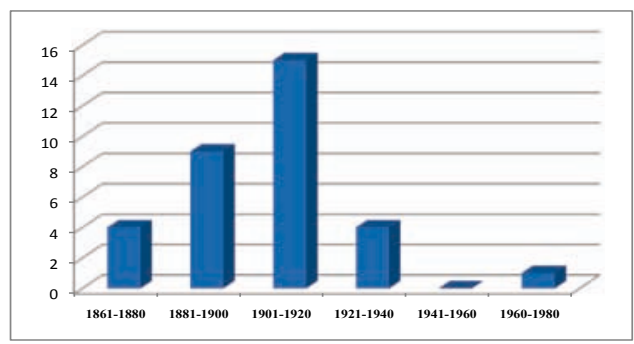

Fuente: Organizaciones colegiales provinciales y bibliografía referenciada. Elaboración Propia.

Debemos considerar y hacer hincapié en que los contenidos documentales conservados en estas asociaciones, en todo el territorio de nuestro país, son de vital importancia para la reconstrucción de la historia profesional, al margen de la iniciativa de la institución mencionada, que nos parece muy importante. Partiendo de la definición de archivo por un lado como, una institución con entidad propia, en 
la que se conserva documentación que ha generado una persona, sociedad u organismo al realizar sus funciones o actividades a lo largo de su existencia como fuente de información para su gobierno. Y de la de archivística por otro, que según T. R. Scchellenberges

(...) la ciencia que trata de los archivos, de su conservación, administración, clasificación, ordenación, interpretación, etcétera; de las colecciones de documentos que en los archivos se conservan como fuente para su conocimiento ulterior y servicio público (Cruz Mundet, 2008, p.57).

Podemos destacar la necesidad que la ordenación de estos repertorios y expedientes personales, generada por la asociación de profesionales de la enfermería en un organismo de su competencia, tiene para la investigación histórica, conocimiento de la misma y como servicio público a los miembros de la disciplina. Pues además de las funciones citadas podemos enumerar las siguientes, teniendo en cuenta que aseguramos con esta acción su atesoramiento para futuras generaciones:

- Clasificar y mantener el orden de los fondos según los principios archivísticos.

- Elaborar instrumentos de descripción de la documentación para hacer más accesible la información contenida en ella, valorando las ventajas que nos ofrecen las nuevas tecnologías.

- Tener en cuenta la legislación existente respecto a los archivos, promulgada tanto a nivel nacional como autonómico.

- Garantizar un equipamiento adecuado del archivo para asegurar la conservación.

- Organización y puesta en servicio para la administración de las oficinas colegiales.

- Iniciar la coordinación con el archivo administrativo, para establecerla transferencia periódica entre ambos de la documentación que deje de ser de uso corriente.

- Aplicar los principios actuales de valoración de los documentos para conservar los de valor y desechar el resto.

- Todas estas funciones han de ser desarrolladas y perfectamente establecidas y garantizadas mediante la creación de un reglamento de funcionamiento del servicio del archivo (Cruz Mundet, 2008, p.93).

No podemos olvidar que todas estas funciones están orientadas al servicio de la administración colegial que es el órgano que genera esta documentación, hacia los profesionales colegiados y hacia la investigación y la difusión cultural (Cruz Mundet, 2008, p.94).

\section{El Archivo del Colegio Oficial de Enfermería}

\section{de Huelva: Cuadro de clasificación.}

La organización del archivo onubense surge ante la necesidad y el planteamiento de la realización de la historia de la institución profesional por la conmemoración de su primer centenario. La primera visita y contacto con el presidente para la petición de los permisos correspondientes en el manejo de los fondos ponen de manifiesto la necesidad de su clasificación, como paso previo a la elaboración de su devenir histórico.

La propuesta de ordenación de los fondos ante los órganos de dirección tuvo una estupenda acogida, máxime cuando la preservación de los mismos se había llevado a cabo por el presidente actual al llegar al cargo y encontrárselos apilados en un pasillo del Colegio. La sensibilidad ante la historia profesional hizo que Gonzalo García Domínguez, distribuyera los AZ y expedientes personales en varias estanterías de la sede colegial aisladas del suelo y la humedad. Al iniciar la organización de la documentación según criterios archivísticos, 
pudimos comprobar que se encontraba en un buen estado de conservación.

Es importante destacar que contábamos con formación histórica pero que carecíamos de la específica para la clasificación archivística. Por tanto, nuestro comienzo sería adquirir estos conocimientos y pedir ayuda a compañeros que tuvieran experiencia archivística suficiente para que nos orientaran en el cometido a realizar.

La primera acción consistía en conocer la organización administrativa del Colegio provincial junto a la revisión de los estatutos, para elaborar el primer índice clasificatorio que no llegaba a ser ni cuadro de clasificación. Este índice tendrá, con el paso del tiempo y la revisión de la documentación, numerosos cambios hasta su estado definitivo, el cual aún no ha llegado. Según Cruz Mundet (2008, p.243) "el cuadro de clasificación no debe ser elaborado apriorísticamente", se ha de partir del conocimiento previo de "la historia, la organización $y$ los procedimientos de la entidad, esto es del contexto que le permita analizar el conjunto de documentos como un todo". Así el archivero con la visión de conjunto podrá identificar las agrupaciones documentales y establecer las diferentes series que compondrán el archivo definitivo.

Conociendo la historia de la institución y la ordenación del archivo administrativo del organismo que lo ha generado, se puede iniciar la revisión de la documentación para su posterior clasificación, siendo fiel al principio de procedencia. Este principio establece que el origen de los documentos debe conservarse según la estructura original, impidiendo que los fondos de un organismo se mezclen con los de otro. La aplicación de este precepto dará lugar a la estructura o partes del cuadro de clasificación (Mendo Carmona, 2004, p.37).

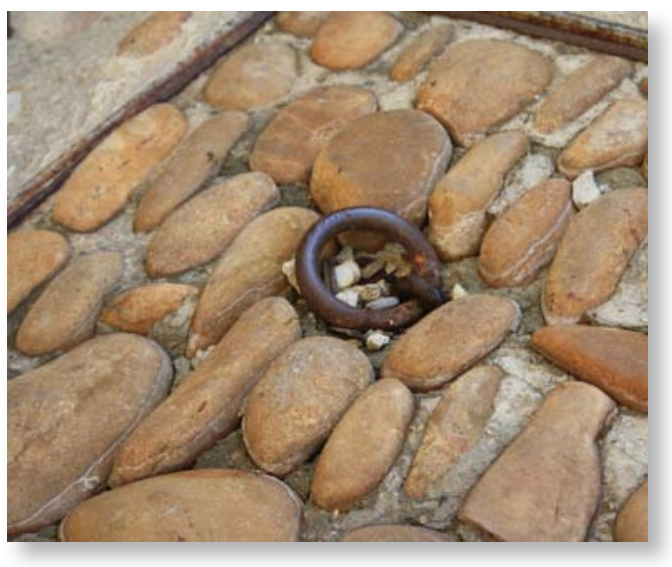

El siguiente paso será la identificación de estas unidades de instalación que se generan progresivamente según vamos descubriendo los fondos revisados. En ella iremos viendo también los documentos gráficos que han se incluirse en un apartado dentro del archivo, para el caso de Huelva aún no hemos acometido ni revisado este tipo de registro.

La revisión de los expedientes personales es de las tareas acometidas en primer lugar en este trabajo archivístico. Se ha creado una base de datos general en la que se han incluido tanto los expedientes históricos como los que se encuentran en activo en el archivo administrativo actual. Este recurso informático cubre tanto las necesidades del histórico como los nuevos requerimientos exigidos desde la Junta de Andalucía. Además evita la posible duplicación de numeración en los casos de traslados de expedientes y posibles vueltas de profesionales entre los distintos Colegios Provinciales del territorio nacional. Por otro lado, nos va a permitir identificar expedientes antiguos con idéntica numeración, procedentes de los tres colegios profesionales anteriores a 1977: el de practicantes, enfermeras y matronas. El volumen de esta Unidad de Instalación es de 131 cajas con un total de 2.488 expedientes, que comprenden una cronología que va desde 1930 a 2010. 
Tras la revisión de todo el conjunto se procederá a la creación definitiva de un cuadro de clasificación lo más claro y sencillo que podamos, para que recoja toda la heterogeneidad de la documentación y de los fondos que comprende.

Una vez que el archivo esté organizado habrá que proceder a crear un sistema de gestión de la documentación del mismo, entre las que estarán la forma de inclusión de nuevos repertorios o traspaso de éstos desde la administración colegial y viceversa, la persona encargada de la guarda y custodia de los fondos, etc. Otro de los aspectos a contemplar será, la gestión de la biblioteca del centro donde encontramos algún que otro ejemplar con un cierto valor histórico, cuya organización ya se ha iniciado. Este tipo de obras, que incluiremos en un "fondo antiguo", serán consultadas en el ámbito de la institución, prohibiendo su salida fuera de la misma, los préstamos ordinarios de los libros tendrán su registro y control correspondiente.

El futuro de todos los archivos debe ser la informatización y digitalización de los fondos que comprende, para el acceso a través de internet en la página web de los distintos colegios profesionales, disminuyendo el deterioro del documento y favoreciendo el acceso sin tener que ir al lugar físico en el que se encuentra el archivo. Otra propuesta de futuro puede ser la integración de todos los archivos colegiales de las distintas provincias a través de una asociación, que podría ser el Consejo General de Colegios Oficiales de Enfermería de España, en nuestro caso, para el acceso a la investigación por los miembros de nuestra disciplina prioritariamente y de otras áreas de conocimiento tanto en el ámbito de la salud como de otras como la historia por citar alguna.
Damos por supuesto, que es nuestra tarea la difusión y promoción de la existencia de los archivos para su conocimiento y función cultural. Esta acción divulgativa puede realizarse a través de folletos publicitarios, boletines informativos, medios de comunicación, la organización de exposiciones tanto permanentes como temporales u otro tipo de eventos, que pueden contribuir al conocimiento por la ciudadanía de la enfermería y su ámbito profesional (Cruz Mundet, 2008, pp.368 y ss.).

\section{CONCLUSIONES}

La organización de los archivos colegiales no es una tarea fácil, sobre todo cuando la documentación puede estar en mal estado o apilada como papeles inservibles, pero nos corresponde a nosotros, los profesionales de enfermería el velar porque esa parte de nuestra historia no se pierda. Es nuestra responsabilidad ponerla al servicio de la comunidad disciplinar para que pueda escribirse el capítulo de la asociación profesional, que aunque no siempre ha estado en consonancia con lo que pensábamos que podía realizar como institución, si pertenece a nuestro devenir histórico. Es un capítulo que hemos de escribir en un periodo en el que se ha llegado a las más altas cotas profesionales en cuanto a la formación disciplinar en la Universidad. En este sentido, el nuevo contexto en el que se enmarcan los archivos, lejos de atesorar sus papeles, los ponen a disposición de todo el público gracias a las nuevas tecnologías y hacen más accesible a todos, la historia y la memoria. La era digital nos puede permitir y de hecho permite el acceso a muchos archivos, infinidad de documentos, otras historias, otros recuerdos y otras memorias. La organización de los archivos de los Colegios Provinciales de Enfermería es una tarea fundamental para poner al servicio de la 
comunidad profesional esta fuente de estudio que no podemos perder para la construcción de nuestra historia.

\section{NOTAS ACLARATORIAS}

${ }^{1}$ Sobre historia de los Colegios Provinciales véase:

- Amezcua, M., Germán, C., Heierle, C. y Del Pozo, M.C. (1994). Sanidad y colectividad sanitaria en Almería. El Colegio de Practicantes (1885-1945). Granada: Colegio de Enfermería de Almería.

- Amezcua, M., Germán, C., Heierle, C. y Del Pozo, M.C. (2006). Apuntes históricos de Gipuzkoa. Practicantes, Matronas y Enfermeras (1904-2004). Donostia- San Sebastián: Colegio de Enfermería de Guipuzcoa.

- Barreiro Pereira, P. y Bermejo, J.M. (2009). El Colegio de Enfermería de Madrid. Cien años de Historia. Madrid: Colegio de Enfermería de Madrid.

- Cortel Espeleta, I., Fuertes Redón, R. y Atienza Salvador, J. (2009). Enfermería turolense, más de cien años de historias. Teruel: Colegio de Enfermería de Teruel.

- Ferreiro Ardións, M. y Lezaun Valdubiesco, J. (2008). Historia de la Enfermería en Álava. Álava: Colegio de Enfermería de Álava.

- Gallardo Moraleda, C., Jaldón García, E. y Villa GarcíaNoblejas, V. (1998). La Enfermería Sevillana. El colegio y su historia 1900-1930. Sevilla: Colegio de Enfermería de Sevilla.

- Gallardo Moraleda, C. y Villa García-Noblejas, V. (1998). La Enfermería Sevillana. El colegio y su historia 19311960. Sevilla: Colegio Oficial de Enfermería de Sevilla.

- Gallardo Moraleda, C. y Villa García-Noblejas, V. (2002). La Enfermería Sevillana. El colegio y su historia 19611990. Sevilla: Colegio Oficial de Enfermería de Sevilla.
${ }^{2}$ Evitamos hacer la referencia a la página web para no herir susceptibilidades, lo más lejos de nuestra intención, pues la traemos como ejemplo aclaratorio del discurso del trabajo que describimos en estas páginas.

\section{BIBLIOGRAFÍA}

- (2003). Actos de celebración del Centenario del Colegio de Enfermería de Valencia (1903-2003). 100 años de unión. Enfermería Integral 65, 4-7.

- (2010). Valores profesionales: 125 años del Colegio de Enfermería de Almería. Enfermería Facultativa (Ed. Andalucía), 133.

- (4 de junio de 2010). El colegio de Enfermería, Pascual Parrilla y Fecoam recibirán las Medallas de Oro de la Región. Diario La Verdad, Recuperado de: http://www. laverdad.es/murcia/hemeroteca/index.php?action=sear ch\&format $=$ noticias\&query $=\mathrm{El}+$ colegio $+\mathrm{de}+$ Enfermer $\% \mathrm{EDa} \% 2 \mathrm{C}+$ Pascual+Parrilla $+\mathrm{y}+\mathrm{Fecoam}+$ recibir $\%$ E1n + las+Medallas+de+Oro+de+la+Regi\%F3n.

- Amezcua, M., Germán, C., Heierle, C. y Del Pozo, M.C. (1994). Sanidad y colectividad sanitaria en Almería. El Colegio de Practicantes (1885-1945). Granada: Colegio Oficial de Enfermería.

- Barreiro Pereira, P. y Bermejo, J.M. (2009). El Colegio de Enfermería de Madrid. Cien años de Historia. Madrid: Colegio de Enfermería de Madrid.

- Cruz Mundet, J. R. (2008). Manual de Archivística. Madrid.

- Expósito González, R. (2010). El congreso español de cirujanos, ministrantes, practicantes y dentistas de 1855. Enfermería de Ciudad Real, 30-33.

- Mendo Carmona, C. (2004). Consideraciones sobre el método en Archivística. Documenta \& Instrumenta, 35-46.

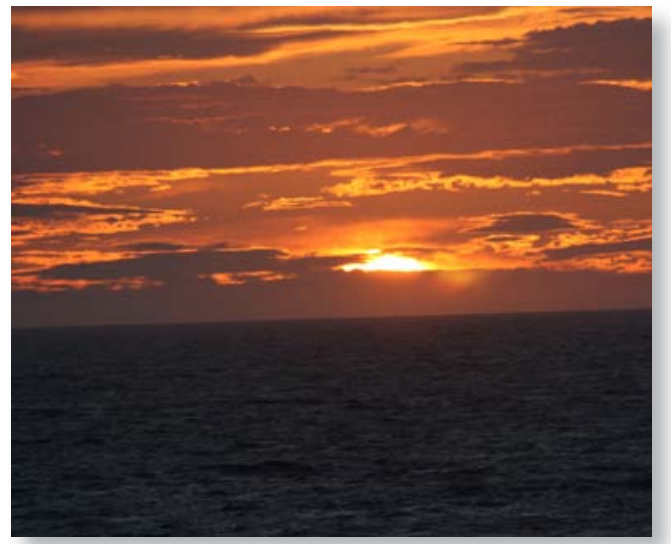

